\title{
On an extension of a theorem of Schur
}

\author{
by \\ Pierre Bornsztein (Antony)
}

1. Introduction. A well-known theorem of I. Schur ([3], [4]) states that "If the positive integers less than $n$ ! $e$ are partitioned into $n$ classes in any way, then $x+y=z$ can be solved in integers (not necessarily distinct) within one class".

For example, in the case $n=2$, the above theorem may be used for $\{1,2,3,4,5\}$. Let us consider the partition $A_{1}=\{1,2\}$ and $A_{2}=\{3,4,5\}$. It is clear that $x+y=z$ has a solution within $A_{1}$. But such a partition does not give a solution if we restrict to triplets $(x, y, z)$ of pairwise distinct integers. Note that if $x+y=z$ with $x, y, z \neq 0$, only $x$ and $y$ may be equal.

Sierpiński [4] has proved that a solution in distinct integers is certain if we replace the upper bound $[n ! e]$ by $2^{[n ! e]}$, and Irving ([1], [2]) improved this result with the bound $\left[\frac{1}{2}(2 n+1) e \cdot n !\right]+2$ (where, as usual, $[x]$ denotes the greatest integer less than or equal to $x$ ).

The purpose of this paper is to prove the following theorem:

Theorem. Let $n \geq 2$ be an integer. If the set $A=\{1,2, \ldots,[n ! \cdot n e]+1\}$ is divided into $n$ classes in any way, then at least one of the classes contains two different numbers and their sum.

2. Preliminaries. Let $n \geq 2$ be an integer. We define the finite sequence $\left(\alpha_{k}\right)$ for $k=1, \ldots, n+1$ by

$$
\alpha_{k}=k+n \cdot(k-1) ! \sum_{i=0}^{k-2} \frac{1}{i !},
$$

with the convention $\sum_{i=p}^{q}=0$ when $p>q$. In particular $\alpha_{1}=1$. Moreover, it is clear that $\alpha_{k} \in \mathbb{N}^{*}$ for each $k$.

Lemma 1. We have $\alpha_{n+1}=[n ! \cdot n e]+1$.

2000 Mathematics Subject Classification: Primary 11B75. 
Proof. We have

$$
\alpha_{n+1}=n+1+n \cdot n ! \sum_{i=0}^{n-1} \frac{1}{i !}=1+n \cdot n ! \sum_{i=0}^{n} \frac{1}{i !},
$$

that is,

$$
\alpha_{n+1}=1+n \cdot n ! e-n \cdot n ! R_{n} \quad \text { with } \quad R_{n}=\sum_{i=n+1}^{\infty} \frac{1}{i !} .
$$

But

$$
\begin{aligned}
R_{n} & =\frac{1}{n !}\left(\frac{1}{n+1}+\frac{1}{(n+1)(n+2)}+\ldots\right)<\frac{1}{n !}\left(\frac{1}{n+1}+\frac{1}{(n+1)^{2}}+\ldots\right) \\
& =\frac{1}{n \cdot n !} .
\end{aligned}
$$

Thus $\alpha_{n+1}<1+n \cdot n ! e<\alpha_{n+1}+1$. Since $\alpha_{n+1}$ is an integer, we deduce that $\alpha_{n+1}=[n ! \cdot n e]+1$.

LEMma 2. For each $k \geq 1$, we have $\alpha_{k+1}=k\left(\alpha_{k}+n-k+1\right)+1$.

Proof. Let $k \geq 1$. We have

$$
\frac{\alpha_{k+1}-1}{k}=1+n \cdot(k-1) ! \sum_{i=0}^{k-1} \frac{1}{i !}=1+n+n \cdot(k-1) ! \sum_{i=0}^{k-2} \frac{1}{i !} .
$$

Thus $\left(\alpha_{k+1}-1\right) / k=1+n+\alpha_{k}-k$. The conclusion follows.

3. Proof of the Theorem. Take partition of $A=\left\{1, \ldots, \alpha_{n+1}\right\}$ into $n$ pairwise disjoint classes. For $E \subset A$ and $a \in A$, define $\Delta_{a}(E)=\{x-a \mid$ $x \in E$ and $x>a$ \}. Then we consider the following algorithm:

(1) Define $S$ to be the set of all the classes of the decomposition.

(2) Choose one of the classes in $S$ with the maximum number of elements. Denote it by $A_{1}$. Set $S=S-\left\{A_{1}\right\}$.

(3) Define $E_{1}=A_{1}, a_{1}=\min E_{1}, F_{1}=\Delta_{a_{1}}\left(E_{1}\right)-\left\{a_{1}\right\}$.

(4) If $F_{1} \cap A_{1} \neq \emptyset$ then stop. Otherwise, set $p=1$ and continue.

(5) Choose an element of $S$ which contains the maximum number of elements from $F_{p}$. Denote it by $A_{p+1}$. Set $S=S-\left\{A_{p+1}\right\}$.

(6) Define

$$
\begin{gathered}
E_{p+1}=A_{p+1} \cap F_{p}, \quad a_{p+1}=\min E_{p+1}, \\
F_{p+1}=\Delta_{a_{p+1}}\left(E_{p+1}\right)-\left\{\sum_{i=0}^{j} a_{p+1-i} \mid j=0,1, \ldots, p\right\} .
\end{gathered}
$$

(7) If $F_{p+1} \cap\left(\bigcup_{i=1}^{p} A_{i}\right) \neq \emptyset$ then stop. Otherwise, continue.

(8) Set $p=p+1$. Go to (5). 
Claim 1. For each $p \in\{1, \ldots, n\}$, if $E_{p}$ is constructed by the algorithm then:

- $E_{p}$ contains at least $\alpha_{n-p+1}+p+1$ elements.

- $F_{p}$ is constructed, and it contains at least $\alpha_{n-p+1}$ elements.

Proof. By induction on $p$. For $p=1$, from the pigeon-hole principle, $A_{1}$ contains at least

$$
\frac{\alpha_{n+1}}{n}=\frac{1}{n}+n ! \sum_{i=0}^{n} \frac{1}{i !}
$$

elements. Thus, $E_{1}=A_{1}$ contains at least

$$
1+n ! \sum_{i=0}^{n} \frac{1}{i !}=\alpha_{n}+2
$$

elements (from Lemma 2). Moreover, $\alpha_{n}+2 \geq 2$, thus $a_{1}$ is well defined. And $F_{1}$ is constructed: we form the differences in (3) (which decreases the number of elements by 1 ), and delete $a_{1}$ (if necessary). Thus $F_{1}$ contains at least $\alpha_{n}$ elements.

Let $p$ be a fixed integer with $1 \leq p<n$. Suppose that the conclusion holds for the value $p$ and that $E_{p+1}$ is constructed by the algorithm. Then, by the induction hypothesis, $F_{p}$ contains at least $\alpha_{n-p+1}$ elements, none of which belongs to $\bigcup_{i=1}^{p} A_{i}$ (otherwise the algorithm would have stopped from (7), and $E_{p+1}$ would not have been constructed). From the pigeon-hole principle and Lemma $2, A_{p+1}$ contains at least

$$
\frac{\alpha_{n-p+1}}{n-p}=\alpha_{n-p}+p+1+\frac{1}{n-p}
$$

elements from $F_{p}$. It follows that $E_{p+1}$ contains at least $\alpha_{n-p}+p+2$ elements. Moreover, $\alpha_{n-p}+p+2 \geq 2$, thus $a_{p+1}$ is well defined, and $F_{p+1}$ is constructed: we form the differences and make the deletions (if necessary, and not more than $p+1)$. Then $F_{p+1}$ contains at least $\alpha_{n-p}$ elements, which ends the induction, and completes the proof of the claim.

REMARK. The claim ensures that there will be no problem at step (6) of the algorithm.

CASE 1: The algorithm stops at (4). Then there exists $b \in F_{1} \cap A_{1}$. Thus $b=a_{i}-a_{1}$ for some $a_{i} \in A_{1}$, and $b \neq a_{1}$ since $a_{1} \notin F_{1}$. Thus $b+a_{1}=a_{i}$ in $A_{1}$, and the conclusion of the Theorem holds.

CASE 2: The algorithm stops at (7) with $p<n$. First note that it did not stop before.

Claim 2. For each $k \in\{1, \ldots, p\}$, the number $X_{k}=a_{p}+a_{p-1}+\ldots+a_{k}$ belongs to $E_{k}$. 
Proof. By (descending) induction. For $k=p$, we directly have $X_{p}=$ $a_{p} \in E_{p}$. Let $k \in\{2, \ldots, p\}$ be fixed. Suppose that $X_{k} \in E_{k}$. Since $E_{k} \subset$ $F_{k-1} \subset \Delta_{a_{k-1}}\left(E_{k-1}\right)$, there exists $x \in E_{k-1}$ such that $X_{k}=x-a_{k-1}$. Thus $X_{k-1}=X_{k}+a_{k-1}=x$ belongs to $E_{k-1}$. This ends the induction and completes the proof.

Since the algorithm has stopped at (7), there exists $k \in\{1, \ldots, p\}$ such that $F_{p} \cap A_{k} \neq \emptyset$. Then $b=b_{i_{1}}-a_{p} \in F_{p} \cap A_{k}$ for some $b_{i_{1}} \in E_{p}$.

If $k=p$, we have $b \in F_{p}$. Then, by construction, $b \neq a_{p}$. Thus $b_{i_{1}}=b+a_{p}$ in $A_{p}$, and the conclusion of the Theorem holds.

If $k<p$, then $b_{i_{1}} \in E_{p} \subset F_{p-1}$. It follows that

$$
\begin{array}{ll}
b_{i_{1}}=b_{i_{2}}-a_{p-1} & \text { for some } b_{i_{2}} \in E_{p-1} \subset F_{p-2}, \\
b_{i_{2}}=b_{i_{3}}-a_{p-2} & \text { for some } b_{i_{3}} \in E_{p-2} \subset F_{p-3}, \\
\cdots & \\
b_{i_{p-k}}=b_{i_{p-k+1}}-a_{k} & \text { for some } b_{i_{p-k+1}} \in E_{k} \subset A_{k} .
\end{array}
$$

Summing, we obtain

$$
b=b_{i_{p-k+1}}-\sum_{i=k}^{p} a_{i}=b_{i_{p-k+1}}-X_{k} .
$$

But, from Claim 2, we have $X_{k} \in E_{k} \subset A_{k}$. And, since $b \in F_{p}$, we deduce that $b \neq X_{k}$ (see step (6)). It follows that $b+X_{k}=b_{i_{p-k+1}}$ in $A_{k}$, and the conclusion of the Theorem holds.

CASE 3: The algorithm does not stop until $p=n$ in (8). Then $A_{n}, E_{n}, F_{n}$ are constructed by the algorithm. From Claim $1, F_{n}$ contains at least $\alpha_{1}=1$ elements. Thus $F_{n} \neq \emptyset$ and

$$
F_{n} \cap\left(\bigcup_{i=1}^{n} A_{i}\right)=F_{n} \cap A \neq \emptyset .
$$

We deduce that the algorithm stops at (7). The reasoning used in Case 2 may be repeated word for word, and the conclusion of the Theorem holds. Thus the proof is complete.

4. Remarks. Following Sierpiński [4], given a natural number $k$, denote by $n(k)$ the least natural number $n$ with the following property: if the numbers $1, \ldots, n$ are divided into $k$ classes, then at least one class contains two different numbers together with their sum.

Then according to Walker [6]

$$
n(1)=3, \quad n(2)=9, \quad n(3)=24, \quad n(4)=67, \quad n(5)=197 .
$$

Also, see [5], p. 440,

$$
n(k) \geq 1+315^{(k-1) / 5} .
$$


Thus

$$
315^{(k-1) / 5}+1 \leq n(k) \leq[k ! \cdot k e]+1 .
$$

\section{References}

[1] R. K. Guy, Unsolved Problems in Number Theory, 2nd ed., Springer, 1994; E11, 209-211.

[2] R. W. Irving, An extension of Schur's theorem on sum-free partitions, Acta Arith. 25 (1973), 55-63.

[3] I. Schur, Über die Kongruenz $x^{m}+y^{m}=z^{m}(\bmod p)$, Jahresber. Deutsch. Math.Verein. 25 (1916), 114-117.

[4] W. Sierpiński, Elementary Theory of Numbers, North-Holland, 1987, 440-443.

[5] - Theory of Numbers, part 2, PWN, Warszawa, 1959 (in Polish).

[6] G. W. Walker, Solution to the problem E985, Amer. Math. Monthly 59 (1952), 253.

50 rue Prosper Legouté

92160 Antony, France

E-mail: pbornszt@club-internet.fr 